\title{
Classification of real analytic Levi flat hypersurfaces of 1-concave type in Hopf surfaces
}

\author{
Takeo Ohsawa
}

\begin{abstract}
A compact Levi flat hypersurface in a complex manifold is said to be of $q$ concave type if it admits a neighborhood system consisting of $q$-concave manifolds in the sense of Andreotti and Grauert. The real analytic Levi flat hypersurfaces of 1-concave type in Hopf surfaces are classified.
\end{abstract}

\section{Introduction}

The study of smooth real hypersurfaces in complex manifolds was first initiated by Poincaré $[\mathrm{P}]$, who showed that the biholomorphic equivalence theorem that Riemann $[R]$ had asserted in his thesis does not hold true for the smooth domains in $\mathbf{C}^{2}$. The equivalence problem between real hypersurfaces, which was naturally evoked by Poincaré's discovery, has been studied particularly in detail for the boundary of the domains of holomorphy. The initially important invariant is the Levi form (see $[\mathrm{L}],[\mathrm{Kr}],[\mathrm{Li}]$ ), and deep results have been obtained for the boundaries of strongly pseudoconvex domains, on which the Levi form is by definition everywhere positive definite (see $[\mathrm{T}],[\mathrm{CM}],[\mathrm{F}]$ ). On the other hand, total degeneracy of the Levi form defines the class of Levi flat hypersurfaces. Since they are locally equivalent to each other, only the global questions are of interest from the viewpoint of equivalence theory. Although not so much is known about them, it has been gradually understood how the geometry of the ambient manifold is related to the analytic structure of the neighborhoods and the complements of the Levi flat hypersurfaces (see [G1], [G2], [LN], [N], [S1], [Oh1], [Oh4], [Oh3], [Oh5], [B1], [B2], [A]). A question which puzzles many people at present is whether or not the complex projective plane admits a Levi flat hypersurface (see $[\mathrm{C}]$ ). Although there exist several papers asserting the nonexistence of such things, [S2] and [Oh2], for instance, the problem has not yet surrendered to any of them.

In this situation, Levenberg and Yamaguchi [LY] studied real analytic Levi flat hypersurfaces in Hopf surfaces which do not bound Stein domains. Here

Kyoto Journal of Mathematics, Vol. 54, No. 3 (2014), 547-553

DOI $10.1215 / 21562261-2693433$, (C) 2014 by Kyoto University

Received January 23, 2013. Revised April 24, 2013. Accepted May 7, 2013.

2010 Mathematics Subject Classification: Primary 32D15; Secondary 32E40. 
they restricted themselves to the surfaces $\mathcal{H}(a, b)=\left(\mathbf{C}^{2}-\{0\}\right) / \Gamma_{a, b}$, where $\Gamma_{a, b}$ denotes the infinite cyclic subgroup of $\mathrm{GL}(2, \mathbf{C})$ generated by the transformation $(z, w) \mapsto(a z, b w)$ with $|a|,|b|>1$. As an application of their theory, they showed that the domain $\left\{(z, w) \cdot \Gamma_{a, b} \mid z \in \mathbf{C}, \operatorname{Im} w>0\right\}$ in $\mathcal{H}(a, b)$ with $b \in \mathbf{R}$, which is bounded by a Levi flat hypersurface $\left\{(z, w) \cdot \Gamma_{a, b} \mid z \in \mathbf{C}, \operatorname{Im} w=0\right\}$, is Stein. The purpose of the present article is to show that any real analytic Levi flat hypersurface bounding a Stein domain in a Hopf surface is essentially of this type. More precisely, we shall show the following.

\section{THEOREM 1.1}

Let $\mathcal{H}=\mathcal{H}(a, b, \lambda, m)=\left(\mathbf{C}^{2}-\{0\}\right) / \Gamma$ be a primary Hopf surface (see Section 3), and let $X \subset \mathcal{H}$ be a real analytic Levi flat hypersurface of 1-concave type. Then one of the following is true:

(1) $m=1$ and $X$ is of Nemirovski type (see Section 4);

(2) $m \geq 2, \lambda \neq 0$ and $X$ is of generalized Nemirovski type (see Section 4).

Since any Hopf surface is a quotient of a primary Hopf surface by a fixed point free action of a finite group, the above classification is actually complete for the real analytic Levi flat hypersurfaces of 1-concave type in any Hopf surface. The proof of Theorem 1.1 is a straightforward consequence of a well-known fact that the envelope of meromorphy of a domain in a Stein manifold coincides with its envelope of holomorphy, combined with an observation that the projectivized holomorphic tangent bundle of any primary Hopf surface admits a nonconstant meromorphic function (for the details, see the arguments below).

\section{Preliminaries}

Let $M$ be a connected complex manifold of dimension $n \geq 2$, and let $X \subset M$ be a compact real analytic Levi flat hypersurface. Let $T X$ and $T M$ be the tangent bundles of $X$ and of $M$, respectively. Let $T^{1,0} M$ be the holomorphic tangent bundle of $M$, and let $T^{1,0} M \mid X$ be its restriction to $X$. We put $T^{1,0} X=\left(T^{1,0} M \mid\right.$ $X) \cap(T X \otimes \mathbf{C})$. For any complex vector bundle $E$ over $M, \mathbf{P}(E)$ will stand for its projectivization. Let $\left(T^{1,0} M\right)^{*}$ denote the dual bundle of $T^{1,0} M$. Then $T^{1,0} X$ naturally defines a real analytic section of $\mathbf{P}\left(\left(T^{1,0} M\right)^{*}\right)$ over $X$, say, $\sigma$. If $\operatorname{dim} M=2, \sigma$ naturally induces a real analytic section of $\mathbf{P}\left(T^{1,0} M\right)$ over $X$.

\section{LEMMA 2.1}

If $M$ is compact and $M-X$ is Stein, there exists a meromorphic section $\tilde{\sigma}$ of $\mathbf{P}\left(\left(T^{1,0} M\right)^{*}\right)$ over $M$ such that $\tilde{\sigma} \mid X=\sigma$.

\section{Proof}

Since $\sigma$ is real analytic, one can find a neighborhood $U \supset X$ and a holomorphic section $\sigma_{U}$ of $\mathbf{P}\left(\left(T^{1,0} M\right)^{*}\right) \mid U$ such that $\sigma_{U} \mid X=\sigma$. Since $M-X$ is Stein, holomorphic vector bundles and their projectivizations are holomorphically 
embeddable into complex projective spaces. Therefore, since projective spaces are bimeromorphically equivalent to products of Riemann spheres, $\sigma_{U}$ is meromorphically extendable to $M$.

In what follows we shall denote the extension $\tilde{\sigma}$ of $\sigma$ by $\sigma_{X}$, which will be naturally identified with a meromorphic section of $\mathbf{P}\left(T^{1,0} M\right)$ if $\operatorname{dim} M=2$.

We recall here that a complex manifold $\Omega$ is said to be $q$-concave if there exists an exhaustion function $\varphi: \Omega \rightarrow(0,1]$ of class $C^{2}$ such that the Levi form of $\varphi$ has at most $q-1$ nonpositive eigenvalues outside a compact subset of $\Omega$ (see $[A G])$.

\section{DEFINITION 2.1}

We say that $X$ of 1 -concave type if $X$ admits a $q$-concave neighborhood basis.

We note that "1-concave type" is an intrinsic property of $X$ as a CR manifold if $X$ is real analytic.

\section{Meromorphic functions on projectivized bundles}

Let $\mathcal{H}$ be a Hopf surface, which is by definition a compact complex surface whose universal covering space is biholomorphically equivalent to $\mathbf{C}^{2}-\{0\}$. Note that if $X$ is a Levi flat hypersurface in $\mathcal{H}$, then $X$ is of 1-concave type if and only if $M-X$ is Stein, because Hopf surfaces contain no exceptional curves. Indeed, it is easy to see that compact quotients of domains in $\mathbf{C}^{2}$ with algebraic dimension $\leq 1$ can contain only smooth elliptic curves of self-intersection zero as complex analytic subsets of codimension one. It is known by Kodaira $[\mathrm{K}]$ that $\mathcal{H}$ is covered by a primary Hopf surface, that is, there exists an automorphism $\tau$ of $\mathbf{C}^{2}-\{0\}$ of the form $\tau(z, w)=\left(a z+\lambda w^{m}, b w\right)\left(|a| \geq|b|>1, \lambda \in \mathbf{C}, m \in \mathbf{N}\right.$, and $a=b^{m}$ if $\lambda \neq 0)$ such that the quotient $\mathcal{H}(a, b, \lambda, m)$ of $\mathbf{C}^{2}-\{0\}$, by the action of the infinite cyclic group $\Gamma$ generated by $\tau$, is a finite unramified cover of $\mathcal{H}$. It is known that $\mathcal{H}(a, b, 0,1)$ admits a nonconstant meromorphic function if and only if $a^{\mu}=b^{\nu}$ holds for some $\mu, \nu \in \mathbf{N}$. In contrast to this, the following is true for all $\mathcal{H}$.

\section{THEOREM 3.1}

$\mathbf{P}\left(T^{1,0} \mathcal{H}\right)$ admits a nonconstant meromorphic function.

Proof

Clearly it suffices to show the assertion for $\mathcal{H}(a, b, \lambda, m)$. From the definition, $T^{1,0} \mathcal{H}(a, b, \lambda, m)$ is equivalent to the quotient of $\mathbf{C}^{2}-\{0\} \times \mathbf{C}^{2}$ by the action of the group generated by $(\tau, d \tau)$. Letting $((z, w),(\xi, \eta))$ be the coordinate of $\mathbf{C}^{2} \times \mathbf{C}^{2}$, where $(\xi, \eta)$ represents the vector $\xi \partial / \partial z+\eta \partial / \partial w$, one has

$$
d \tau(\xi, \eta)=\left(a \xi+m \lambda w^{m-1} \eta\right) \partial / \partial z+b \eta \partial / \partial w .
$$


Therefore $\xi w / \eta z$ is invariant under $(\tau, d \tau)$ if $\lambda=0$, and so is the function

$$
\xi / \eta w^{m-1}-m z / w^{m}
$$

otherwise.

\section{Levi flat hypersurfaces of 1-concave type in Hopf surfaces}

Some of the Levi flat hypersurfaces of 1-concave type in torus bundles over compact Riemann surfaces have been constructed in [N], [Oh1], and [Oh5]. To define a class of Levi flat hypersurfaces in Hopf surfaces, we shall recall the description of such hypersurfaces in [Oh5], adjusting the notation in the present situation.

Let $p: \mathbf{C}^{2}-\{0\} \rightarrow \mathbf{C P}^{1}$ be the natural projection, and let $\pi: \mathbf{C}^{2}-\{0\} \rightarrow \mathcal{H}$ be the universal covering map. Let $\zeta=z / w$ be the inhomogeneous coordinate of $\mathbf{C P}^{1}$, and set

$$
\begin{aligned}
& U_{+}=\{\zeta|0 \leq| \zeta \mid<\infty\}, \\
& U_{-}=\{\zeta|0<| \zeta \mid \leq \infty\} .
\end{aligned}
$$

Then, with respect to any pair of meromorphic 1 -forms $\omega_{ \pm}$on $U_{ \pm}$satisfying

$$
\omega_{+}-\omega_{-}=d \log \zeta \quad \text { on } U_{+} \cap U_{-}
$$

parallel transports of points in $\mathbf{C}^{2}-\{0\}$ are defined over the real differentiable paths in $\mathbf{C P}^{1}$ that avoid the poles of $\omega_{ \pm}$. Namely, over such curves $\gamma:[0,1] \rightarrow U_{ \pm}$, the transports are given in the fiber coordinates by

$$
w \mapsto w \exp \int_{\gamma} \omega_{+} \quad \text { on } p^{-1}\left(U_{+}\right)
$$

and

$$
z \mapsto z \exp \int_{\gamma} \omega_{-} \quad \text { on } p^{-1}\left(U_{-}\right)
$$

Here $w$ and $z$ are used as the fiber coordinates of $p^{-1}\left(U_{+}\right)$and $p^{-1}\left(U_{-}\right)$, respectively.

Let $P_{\infty}$ be the union of the set of poles of $\omega_{+}$and $\omega_{-}$. Then, fixing a point $\zeta_{0}$ in $\mathbf{C P}^{1}-P_{\infty}$, the transports of any closed real 1-dimensional submanifold $S$ in $p^{-1}\left(\zeta_{0}\right)$ yields a Levi flat hypersurface in $p^{-1}\left(\mathbf{C P}^{1}-P_{\infty}\right)$, if the parallel transports over closed paths in $\mathbf{C P}^{1}-P_{\infty}$ leave $S$ invariant.

If $S$ satisfies this invariance condition, we shall denote by $X_{S}$ the hypersurface in $p^{-1}\left(\mathbf{C P}^{1}-P_{\infty}\right)$ obtained by the parallel transports with respect to $\omega_{ \pm}$. Then the closure $\overline{X_{S}}$ of $X_{S}$ in $\mathbf{C}^{2}-\{0\}$ becomes a smooth Levi flat hypersurface if and only if certain conditions on the poles of $\omega_{ \pm}$as described in [Oh5] are satisfied. Existence questions of such $\omega_{ \pm}$aside, hypersurfaces $X$ in $\mathcal{H}$ such that $\pi^{-1}(X)=\overline{X_{S}}$ for some $S$ shall be said to be of Nemirovski type.

More generally, if there exist a holomorphic map $\tilde{p}: \mathbf{C}^{2}-\{0\} \rightarrow \mathbf{C P}^{1}$ of the form $\tilde{p}(z, w)=(z+f(w): w)$ for some polynomial $f(w)$, and meromorphic 1-forms $\omega^{\prime}$ on $U^{\prime}:=\{w \neq 0\}$ and $\omega^{\prime \prime}$ on $U^{\prime \prime}:=\{\tilde{p} \neq 0\}$ satisfying $\omega^{\prime}-\omega^{\prime \prime}=$ $d \log ((z+f(w) / w))$ on $U^{\prime} \cap U^{\prime \prime}$ such that $\pi^{-1}(X)$ is obtained as the closure of 
the parallel transport of a closed real 1-dimensional submanifold of a general fiber of $\tilde{p}$, we shall say that $X$ is of generalized Nemirovski type.

Real analytic Levi flat hypersurfaces of generalized Nemirovski type are of 1-concave type because the distance $\delta$ from $(z, w) \cdot \Gamma$ to $X$, measured fiberwise on $\tilde{p}^{-1}\left(U^{\prime}\right)$ with respect to $|d w| /|w|$, satisfies rank $\partial \bar{\partial} \log \delta=2$ outside the preimages of a finite set. As for an explicit form of such a computation in a more general setting, see [M], for instance.

\section{Proof of Theorem 1.1}

If $\mathcal{H}=\mathcal{H}(a, b, 0,1)$, then the image of $\sigma_{X}$ intersects with the preimages of $\xi w / \eta z$ along level sets of $\xi / \eta$, since $\mathcal{H}$ contains no compact complex curves $C$ such that $p\left(\pi^{-1}(C)\right)=\mathbf{C P}^{1}$. This shows that $X$ is of Nemirovski type. If $\mathcal{H}=\mathcal{H}(a, b, \lambda, 1)$, a similar argument applies for the function $(\xi / \eta-z / w) /(z / w-\lambda)$ instead of $\xi w / \eta z$, so that $X$ is also of Nemirovski type. If $\mathcal{H}=\mathcal{H}(a, b, \lambda, m)$, with $\lambda \neq 0$, $m \geq 2$, and $a=b^{m}$, a similar method also works with respect to the fibration $\tilde{p}: \mathbf{C}^{2}-\{0\} \rightarrow \mathbf{C P}^{1}$, where

$$
\tilde{p}(z, w)=\left(z+c w^{m} / m: w\right)
$$

and $c$ is a constant such that the image of $\sigma_{X}$ is contained in the preimage of $c$ by $\xi / \eta w^{m-1}-m z / w^{m}$.

\section{Remarks}

Responding to a request from the referees, the author would like to add remarks on the Levi flat hypersurfaces in Hopf manifolds of dimension $\geq 3$.

(1) Let $\mathcal{H}^{n}=\mathbf{C}^{n}-\{0\} / \Gamma_{a}$, where $\Gamma_{a}$ denotes the group generated by $z \mapsto a z$ $(|a|>1)$, and let $X \subset \mathcal{H}$ be a real analytic Levi flat hypersurface. Then, by a theorem of Merker and Porten [MP], an argument similar to that in Section 4 above works to describe such $X$ provided that $\mathcal{H}-X$ is $(n-1)$-complete; $X$ bounds a Stein bundle over a Stein domain in $\mathbf{C P}^{n-1}$ unless $X$ is fibered over a Levi flat hypersurface in $\mathbf{C P}^{2}$. Therefore, up to a long-standing open question whose affirmative answer would exclude the latter possibility, they all belong to a class implicitly given by Nemirovski [N].

(2) Let $\mathcal{H}^{(n)}=\mathbf{C}^{n}-\{0\} / \Gamma^{(n)}$, where $\Gamma^{(n)}$ is generated by $z \mapsto\left(2 z_{1}+z_{n}, \ldots\right.$, $\left.2 z_{n-1}+z_{n}, 2 z_{n}\right)$. Then the domain $D_{n}=\left\{z \Gamma^{(n)} \mid z \in \mathbf{C}^{n}-\{0\}, \operatorname{Im} z_{n}>0\right\}$ in $\mathcal{H}^{(n)}$ is bounded by a Levi flat hypersurface. $D_{n}$ is Stein because it is a pseudoconvex Riemann domain over $\mathbf{C}^{n}$ by the map induced from

$$
z \mapsto\left(\exp \left(4 \pi i z_{1} / z_{n}\right), \ldots, \exp \left(4 \pi i z_{n-1} / z_{n}\right), z_{n} 2^{-2 z_{1} / z_{n}}\right),
$$

and $\partial D_{n}$ is of Nemirovski type in a sense similar to that in Section 4 .

Finally, the author would like to comment that the method of this paper works to determine the holomorphic foliations, possibly with singularities, on compact complex surfaces of algebraic dimension $\leq 1$ whose projectivized tangent bundles are a union of the images of meromorphic sections. 
Acknowledgments. The author is grateful to H. Yamaguchi, T. Ueda, and I. Nakamura for stimulating discussions.

\section{References}

[A] M. Adachi, On the ampleness of positive CR line bundles over Levi-flat manifolds, Publ. Res. Inst. Math. Sci. 50 (2014), 153-167. MR 3167582. DOI 10.4171/PRIMS/127.

[AG] A. Andreotti and H. Grauert, Théorème de finitude pour la cohomologie des espaces complexes, Bull. Soc. Math. France 90 (1962), 193-259. MR 0150342.

[B1] M. Brunella, On the dynamics of codimension one holomorphic foliations with ample normal bundle, Indiana Univ. Math. J. 57 (2008), 3101-3113. MR 2492227. DOI 10.1512/iumj.2008.57.3394.

[B2] Codimension one foliations on complex tori, Ann. Fac. Sci. Toulouse Math. (6) 19 (2010), 405-418. MR 2674768.

[C] D. Cerveau, Minimaux des feuilletages algébriques de $\mathrm{CP}(n)$, Ann. Inst. Fourier (Grenoble) 43 (1993), 1535-1543. MR 1275208.

[CM] S. S. Chern and J. Moser, Real hypersurfaces in complex manifolds, Acta Math. 133 (1974), 219-271. MR 0425155.

[F] C. Fefferman, The Bergman kernel and biholomorphic mappings of pseudoconvex domains, Invent. Math. 26 (1974), 1-65. MR 0350069.

[G1] H. Grauert, On Levi's problem and the imbedding of real-analytic manifolds, Ann. of Math. (2) 68 (1958), 460-472. MR 0098847.

[G2] _ Bemerkenswerte pseudokonvexe Mannigfaltigkeiten, Math. Z. 81 (1963), 377-391. MR 0168798.

$[\mathrm{K}] \quad$ K. Kodaira, On the structure of compact complex analytic surfaces, II, Amer. J. Math. 88 (1965), 682-721. MR 0205280.

[Kr] J. Krzoska, Über die natürlichen Grenzen analytischer Funktionen mehrerer Veränderlichen, Ph.D. dissertation, University of Greifswald, Greifswald, Germany, 1933.

[LY] N. Levenberg and H. Yamaguchi, Pseudoconvex domains in the Hopf surface, to appear in J. Math. Soc. Japan, preprint, arXiv:1205.3346v1 [math.CV].

[L] E. E. Levi, Sulle ipersuperficie delle spazi a 4 dimensioni che possone essere frontiera del campo de esistenza di una funzione analitica di due variabli complesse, Ann. Mat. Pura Appl. 18 (1911), 69-79.

[Li] I. Lieb, "Das Levishe problem" in Beiträge zur komplexen Analysis, Bonner Math. Schriften 387, Bonn Univ., Bonn, 2007, 1-34. MR 2446227.

[LN] A. Lins Neto, A note on projective Levi-flats and minimal sets of algebraic foliations, Ann. Inst. Fourier (Grenoble) 49 (1999), 1369-1385. MR 1703092.

[M] K. Matsumoto, "Levi form of logarithmic distance to complex submanifolds and its application to developability" in Complex Analysis in Several 
Variables: Memorial Conference of Kiyoshi Oka's Centennial Birthday, Adv. Stud. Pure Math. 42, Math. Soc. Japan, Tokyo, 2004, 203-207. MR 2087052.

[MP] J. Merker and E. Porten, The Hartogs extension theorem on $(n-1)$-complete complex spaces, J. Reine Angew. Math. 637 (2009), 23-39. MR 2599079. DOI 10.1515/CRELLE.2009.088.

[N] S. Nemirovski, Stein domains with Levi-flat boundaries in compact complex surfaces, Math. Notes 66 (1999), 522-525. MR 1747093.

DOI 10.1007/BF02679105.

[Oh1] T. Ohsawa, A Stein domain with smooth boundary which has a product structure, Publ. Res. Inst. Math. Sci. 18 (1982), 1185-1186. MR 0688952. DOI 10.2977/prims/1195183303.

[Oh2] - Nonexistence of real analytic Levi flat hypersurfaces in $\mathbf{P}^{2}$, Nagoya Math. J. 158 (2000), 95-98. MR 1766572.

[Oh3] A remark on pseudoconvex domains with analytic complements in compact Kähler manifolds, J. Math. Kyoto Univ. 47 (2007), 115-119.

MR 2359103.

[Oh4] On the complement of Levi-flats in Kähler manifolds of dimension $\geq 3$, Nagoya Math. J. 185 (2007), 161-169. MR 2301464.

[Oh5] A A reduction theorem for stable sets of holomorphic foliations on complex tori, Nagoya Math. J. 195 (2009), 41-56. MR 2552952.

[P] H. Poincaré, Les fonctions analytique de deux variables et la représentation conforme, Rend. Circ. Mat. Palermo 23 (1907), 185-220.

[R] B. Riemann, Grundlagen für eine allgemeine Theorie der Functionen einer veränderlichen complexen Grösse, Göttingen, 1851.

[S1] Y.-T. Siu, Nonexistence of smooth Levi-flat hypersurfaces in complex projective spaces of dimension $\geq 3$, Ann. of Math. (2) 151 (2000), 1217-1243. MR 1779568. DOI 10.2307/121133.

[S2] _ symmetric spaces with respect to invariant metrics, Ann. of Math. (2) 156 (2002), 595-621. MR 1933078. DOI 10.2307/3597199.

[T] N. Tanaka, On the pseudo-conformal geometry of hypersurfaces of the space of $n$ complex variables, J. Math. Soc. Japan 14 (1962), 397-429.

MR 0145555.

Graduate School of Mathematics, Nagoya University, Furo-cho, Chikusa-ku, Nagoya 464-8602 Japan; ohsawa@math.nagoya-u.ac.jp 